\title{
Knowledge on sudden unexplained infant death-related safe sleep practices and infant cardiopulmonary resuscitation in pediatric nurses
}

\author{
Jung Ae Cho ${ }^{1}$, Min Sohn ${ }^{2}$, Sangmi Lee ${ }^{3}$, Young Mee Ahn ${ }^{2}$ \\ ${ }^{1}$ Graduate Assistant, Department of Nursing, College of Medicine, Inha University, Incheon; ${ }^{2}$ Professor, Department of Nursing, College of Medicine, Inha \\ University, Incheon; ${ }^{3}$ Associate Professor, Department of Nursing, Dongyang University, Yeongju, Korea
}

Purpose: Sudden unexplained infant death (SUID) is a major contributor to infant mortality, and pediatric nurses have the responsibility to educate parents on SUID-reducing strategies. This study was conducted to measure pediatric nurses' knowledge of SUID-related safe sleep practices (K-SSSP) and infant cardiopulmonary resuscitation (K-ICPR). Methods: In total, 136 pediatric nurses were administered a survey including K-SSSP ( 13 items), K-ICPR (5 items), confidence in K-SSSP education (1 item; 5 points), and other factors relating to SUID experiences or education. Results: The correct answer rates of the K-SSSP and K-ICPR were $62.6 \%$ and $62.5 \%$, retrospectively. The mean score for confidence in K-SSSP education was $2.6 \pm 0.9$. Only 18 nurses $(13.2 \%)$ responded that they educated parents on the content of the K-SSSP, while 76 nurses had received education on SUID. Positive relationships were observed between K-SSSP scores and higher education, between K-ICPR scores and having own child(ren) and clinical experience, and between confidence in K-SSSP education and higher education or having one's own child(ren). Nurses caring for newborns performed more SUID education than nurses working in other units. Conclusion: There is a profound need to implement a systemic educational program on SUID and strategies to reduce SUID for pediatric nurses.

Key words: Knowledge; Child care; Pediatric nurses; Self confidence; Sudden infant death syndrome

\section{Corresponding author Young Mee Ahn \\ https://orcid.org/0000-0003-1404-7849}

Department of Nursing, College of Medicine, Inha University, 100 Inharo, Michuhol-gu, Incheon 22212, Korea

TEL +82-32-860-8207 FAX +82-32-874-5880

E-MAIL aym@inha.ac.kr

*This study was supported by a research grant of the Division of Forensic Medicine at the National Forensic Service (NFS) (2020-Forensic Medicine-07).

*This article was adapted from a thesis by Jung Ae Cho in partial fulfilment of the requirements for the master's degree at Inha University.

Received Sep 18, 2020 Revised Oct 5, 2020 Accepted Oct 9, 2020 (a) This is an Open Access article distributed under the terms of the Creative Commons Attribution NonCommercial License (http://creativecommons.org/licenses/by-nc/4.0/) which permits unrestricted noncommercial use, distribution, and reproduction in any medium, provided the original work is properly cited.

\section{INTRODUCTION}

Sudden unexplained infant death (SUID) refers to unexpected death during sleep in infants younger than 1 year of age [1]. When a SUID case occurs, the cause of death is investigated through an autopsy and field and medical records, and sudden infant death syndrome (SIDS) is diagnosed when the cause of death cannot be determined through the investigation [1]. The exact cause of SUID is not known yet, but according to the triple risk model developed by Filiano and Kinney [2], SUID can occur when intrinsic factors such as the genotype, premature birth, and exposure to cigarette smoke in utero are compounded with external risk factors within 6 months, a critical period for infant development [3].

The SUID rate in South Korea was 0.2 infants per 1,000 births in 2018, and it was the third-highest cause of infant death [4]. The National Forensic Service (NFS) of Korea estimated the SUID rate to be 0.22-0.39 infants per 1,000 births from 13 years of autopsy cases [5]. In United States, the prevalence of SUID was 1.3-1.4 infants per 1,000 births before the 1990s [6]. The American Academy of Pediatrics (AAP) proposed safe sleep and environment management guidelines to reduce the incidence of SUID [7]. In many countries, including 
the United States and Australia, a population-wide education campaign known as the Back-to-Sleep campaign was conducted in the early 1990s to promote sleeping straight on the back. Later, the focus of the campaign expanded to a safe sleep environment, and the name of the campaign was changed to Safe Sleep. As a result of the Safe Sleep campaign [7], the SUID incidence was reduced to 0.35 infants per 1,000 births, suggesting that the campaign was successful [8]. In South Korea, SIDS prevention education has been conducted sporadically $[9,10]$, but has not been regularly implemented through a long-term education campaign. Moreover, the lack of a dedicated private or public organization has led to deficiencies in SUID education and public awareness campaigns. However, since child care and sleep habits are cultural phenomena that vary across countries and ethnicities, overseas campaigns, guidelines, or education materials that are directly imported to South Korea have limited effectiveness and appropriateness.

According to the AAP guideline, a sleeping position on the belly or on the side, soft bedding, swaddling, and overheating constitute unsafe sleep environments, and aspects of the external environment, such as exposure to cigarette smoke, alcohol use of caretakers, and humid and unhygienic living conditions, are also proposed to have direct and indirect effects on the occurrence of SUID [7]. The risk of SUID can be reduced by educating infants' parents about safe sleeping practices and appropriate responses in emergency situations. Infants' parents acquire information related to SUID from medical staff such as nurses and doctors, family members, and the internet [11], and the AAP guidelines also emphasize health professionals' role in educating parents regarding SUID and safe sleeping environments [7]. According to prior research, when parents were not educated about sleeping positions at the hospital nursery, $22 \%$ of mothers of infants put their infants in a straight sleep position on their backs at home, whereas this proportion increased to $70 \%$ after education [12]. Parents who received education on infant cardiopulmonary resuscitation (CPR) when they were discharged from the hospital nursery more efficiently responded to accidents such as infant suffocation [13]. Nurses working in maternal-newborn units provide guidance on safe sleep practices to parents. However, an integrative review of 16 studies on SIDS reported that some nurses still recommended incorrect sleep positioning to mothers due to fear of aspiration [14]. In a correlational study on infant safe sleep practices among nurse practitioners, lack of knowledge and lack of confidence were strongly correlated, which could result in incorrect practices in educating parents on infant safe sleep practices [15]. In order to develop and adopt an educational program on infant safe sleep practices, there is a need to explore what nurses know about SUID-related safe sleep practices, infant CPR, and related factors. This study (1) measured scores on the Knowledge of SUID-Related Safe Sleep Practices (K-SSSP) and Knowledge of Infant Cardiopulmonary Resuscitation (K-ICPR) tools among pediatric nurses and (2) investigated the associations of these knowledge measures with confidence in SUID-related safe sleep practices (SSSP) education and clinical experiences of SSSP and infant cardiopulmonary resuscitation (ICPR).

\section{METHODS}

\section{Study Design}

This descriptive study investigated the associations of the K-SSSP and K-ICPR with confidence in SSSP education and clinical experiences of SSSP and ICPR among pediatric nurses.

\section{Participants}

The target samples of the study were pediatric nurses who worked in departments with infants or children at hospitals. Three secondary or higher-level hospitals in the metropolitan area were selected, in which the levels of medical services and facilities may be similar to each other. We intended to include all nurses who met the criteria from the hospitals through convenience sampling. The specific eligibility criteria were pediatric nurses with more than 6 months of experience who worked in hospital nurseries, neonatal intensive care units (NICU), pediatric wards, pediatric emergency rooms, pediatric outpatient departments, delivery rooms, and obstetric wards. There were approximately 190 pediatric nurses at the study sites. Among them, a total of 142 nurses $(74.7 \%)$ from 11 related wards at three hospitals were identified as eligible participants, and 136 nurses (95.8\%) actually responded to the survey.

\section{Variables}

The main variables in this study were the K-SSSP, K-ICPR, confidence in SSSP education, and clinical experiences of SSSP.

\section{1) Knowledge of SUID-related safe sleep practices(K-SSSP)}

The preliminary items of the K-SSSP were developed based on the SUID-related safe environment guidelines from the $\mathrm{AAP}[7]$ and the domestic and international literature $[4,5,16$, 17]. We reviewed the details of the guidelines and protocols and identified the standard content on safe sleep practices such as positioning, as well as points to emphasize for parents in Korea, such as bed-sharing. A professor of pediatric nursing and two pediatric nurses with more than 7 years of experi- 
ence checked the content validity, relevance, and specificity of the items. The NICU nurses and pediatric nursing professors evaluated the applicability of the wording and the structure of the tools. The final K-SSSP consisted of 13 items on knowledge in two areas: six items on infant safe sleep practices and seven items on SUID. It dealt with safe usage of blankets, swaddle wraps, and bedding (six items), as well as sleeping position, temperature, SUID mechanisms, and the use of monitoring devices (seven items). The items included true/false questions and multiple-choice questions. Each correct response was assigned 1 point and each incorrect response received 0 points. The possible scores ranged from 0 to 13 . The test-retest correlation at 4 months apart showed an intraclass correlation coefficient of .52.

\section{2) Knowledge of infant cardiopulmonary resuscitation (K-ICPR)}

The K-ICPR tool was developed in similar way to the KSSSP. After reviewing infant CPR guidelines by the American Heart Association and the Korean Association of Cardiopulmonary Resuscitation [18,19], 5 items were created to assess knowledge on securing the airway, the ratio of chest compressions to breaths in one-person rescue, the rate and method of chest compressions, and the removal of inhaled foreign bodies. The structure of the items was either true/false or multiplechoice questions and a range of possible scores of 0 to 5 . The validity and reliability of the K-ICPR were assessed in similar way to the K-SSSP. The test-retest correlation at 4 months apart showed an intraclass correlation coefficient of .45.

\section{3) Confidence in SUID-related safe sleep practices education}

Confidence in SSSP education referred to individuals' confidence in educating parents or carrying out practices suggested in the AAP guidelines. It was measured on a 5-point visual analogue scale (VAS). It is widely considered acceptable to apply VAS as tools to measure psychosocial states such as health behavior prediction [20] or pain [21]. The lowest confidence level corresponded to a score of 1 , and the highest confidence level to a score of 5 .

\section{4) Experiences of SUID-related safe sleep practices education}

The experiences of SSSP education were assessed in two areas: the experience of receiving education on SSSP for nurses themselves and the experience of providing education on SSSP to parents. The participants were asked about the presence or absence of these two experiences and the types of education if they reported relevant experiences.

\section{5) General characteristics of participants}

Based on a literature review $[15,22,23]$, variables such as sex, age, marital status, having own child(ren), education level, to- tal clinical experience, and child health care experience were investigated as factors that could impact the K-SSSP, K-ICPR, confidence in SSSP education, and clinical experience.

\section{Data Collection and Procedures}

Prior to data collection, approval was obtained from the Institutional Review Board (IRB) (approval no. 2019-12-032), and participants were recruited after obtaining approval from the head of the nursing department and other relevant departments at each institution. An informed consent form assuring participants of the confidentiality of their responses, the voluntary nature of their decision to participate in the study, and the anonymity of their personal identifiable information was completed by each of the 136 pediatric nurses. Due to the COVID-19 pandemic, the surveys were distributed and the responses were collected by clerical assistants or mailing services, rather than through individual contact with each participant. Data were collected in April 2020.

\section{Data Analysis}

Data collected through the survey were reviewed to check whether the responses were complete, and it was ascertained that the percentage of missing data was less than $10 \%$ for each variable, except pediatric clinical experience, as addressed in Table 1 . The data were analyzed using SPSS for Windows 25.0 (IBM Corp., Armonk, NY, USA). The main variables were demonstrated to have a normal distribution, with skewness of $-0.13 \pm 0.21$ and kurtosis of $-0.54 \pm 0.41$ for the K-SSSP and skewness of $-0.35 \pm 0.21$ and kurtosis of $0.06 \pm 0.41$ for the $\mathrm{K}$ ICPR. The associations among the main variables were analyzing using analysis of variance, Pearson correlation coefficients, the t-test, and the $x^{2}$ test.

\section{RESULTS}

\section{Participants' General Characteristics and Experiences Providing Education on a Safe Sleep Environment}

The average age of the nurses was $33.2 \pm 8.3$ years, their total clinical experience was $8.2 \pm 7.6$ years, and their pediatric clinical experience was $4.3 \pm 5.1$ years (Table 1). More than onethird of participants were married $(32.6 \%)$ or had experience raising children $(36.0 \%)$, and $8.1 \%$ had a master's degree or higher. Fifty-three nurses $(39.0 \%)$ worked in the NICU or nurseries, while $83(61.0 \%)$ worked in pediatric wards, obstetric wards, pediatric intensive care units, pediatric outpatient departments, and delivery rooms. Slightly more than half (55.9\%) of the nurses had received education on SUID, most 
often during their nursing course $(43.4 \%)$. However, only $5.9 \%$ replied that there were guidelines regarding SUID in their wards. Eighteen nurses (13.2\%) responded that they educated parents on a safe sleeping environment; $11.8 \%$ gave verbal instructions, while $0.7 \%$ used written materials.

\section{Pediatric Nurses' SUID-related Knowledge and Confi- dence}

The average K-SSSP score of the pediatric nurses was 8.1, with the lowest score of 4 and highest score of 12 . The average percentage of correct responses was $62.6 \%$. The items for which more than $90 \%$ of the responses were correct were the SUID mechanism (95.6\%), exposure to cigarette smoking $(94.1 \%)$, independent use of bed and bedding (94.1\%), and hard bed surfaces $(91.9 \%)$. The average K-ICPR score was 3.1, with the lowest score of 0 and highest score of 5 . The average percentage of correct responses was $62.5 \%$. Excluding two non-responses, the average score for confidence in SSSP education was 2.6 \pm 0.9 , with the lowest score of 1 and highest score of 5 (Table 2).

Table 1. General Characteristics of Participants $(N=136)$

\begin{tabular}{|c|c|c|}
\hline Characteristics & Categories & $\mathrm{n}(\%)$ or $\mathrm{M} \pm \mathrm{SD}$ \\
\hline Age (year) & & $33.2 \pm 8.3$ \\
\hline Clinical experience (year) & & $8.2 \pm 7.6$ \\
\hline Pediatric nursing experience (year)* & & $4.3 \pm 5.1$ \\
\hline Marital status* & $\begin{array}{l}\text { No } \\
\text { Yes }\end{array}$ & $\begin{array}{l}91(67.4) \\
44(32.6)\end{array}$ \\
\hline Have own child(ren) & $\begin{array}{l}\text { No } \\
\text { Yes }\end{array}$ & $\begin{array}{l}87(64.0) \\
49(36.0)\end{array}$ \\
\hline Level of education & $\begin{array}{l}\text { Associate or bachelor's degree } \\
\text { Master's degree or higher }\end{array}$ & $\begin{array}{l}125(91.9) \\
11(8.1)\end{array}$ \\
\hline Unit of service & $\begin{array}{l}\text { NICU, nursery } \\
\text { Others }^{\dagger}\end{array}$ & $\begin{array}{l}53(39.0) \\
83(61.0)\end{array}$ \\
\hline Received education on SUID & $\begin{array}{l}\text { No } \\
\text { Yes } \\
\text { Origin of information }{ }^{\ddagger}(\mathrm{n}=76) \\
\text { - Nursing course } \\
\text { - Media; nursing continuing education } \\
\text { - Practice guideline } \\
\quad \text { - Professional resource }\end{array}$ & $\begin{array}{c}60(44.1) \\
76(55.9) \\
59(43.4) \\
14(10.3) \\
8(5.9) \\
7(5.1)\end{array}$ \\
\hline Delivery of SSSP education & $\begin{array}{l}\text { No } \\
\text { Yes } \\
\text { Methods } *^{*}(\mathrm{n}=18) \\
\quad \text { - Verbal education } \\
\text { - Use of written materials }\end{array}$ & $\begin{array}{r}118(86.8) \\
18(13.2) \\
16(11.8) \\
1(0.7)\end{array}$ \\
\hline
\end{tabular}

*Missing data were not included; ${ }^{\dagger}$ Pediatric ward $(n=46)$, obstetric ward $(n=21)$, pediatric emergency room $(n=9)$, pediatric outpatient department $(\mathrm{n}=6)$, delivery room $(\mathrm{n}=1) ;{ }^{\dagger}$ Multiple responses possible; NICU, neonatal intensive care unit; SSSP, sudden unexplained infant death related safe sleep practices; SUID, sudden unexplained infant death.

Table 2. Responses to Questions on Knowledge of SUID-related Safe Sleep Practices, Knowledge of Infant Cardiopulmonary Resuscitation, and Confidence in Education on SUID-related Safe Sleep Practices $(N=136)$

\begin{tabular}{llcc}
\hline Variables & Categories & M \pm SD & Min-Max \\
\hline Knowledge of SUID-related safe sleep & - Total knowledge score & $8.1 \pm 1.8$ & $4.0-12.0$ \\
$\quad$ practices & - Correct answer rate (total) & $62.6 \pm 13.7$ & $30.8-92.3$ \\
Knowledge of infant cardiopulmonary & - Total knowledge score & $3.1 \pm 1.0$ & $0.0-5.0$ \\
$\quad$ resuscitation & - Correct answer rate (total) & $62.5 \pm 20.0$ & $29.4-88.2$ \\
Confidence in SSSP education* & & $2.6 \pm 0.9$ & $1.0-5.0$ \\
\hline
\end{tabular}

*Missing data were not included; SSSP, sudden unexplained infant death related safe sleep practices; SUID, sudden unexplained infant death. 


\section{Associations among SUID-related Knowledge, Confi- dence, and Parental Education}

Table 3 displays the results of the analysis of associations between pediatric nurses' knowledge of safe sleep environments and their confidence. Confidence in SSSP education had a weak positive association with the K-SSSP $(\mathrm{r}=.22, p=$ $.012)$ and the K-ICPR ( $r=.23, p=.009)$. A meaningful difference in the K-ICPR was found according to whether they conducted parental education on SSSP $(t=2.25, p=.026)$, but the K-SSSP did not show such a relationship $(\mathrm{t}=1.79, p=.075)$.

\section{Pediatric Nurses' Characteristics and SUID-related Knowledge, Confidence, and Parental Education}

\section{1) Pediatric nurses' characteristics and SUID-related knowledge}

When the K-SSSP was analyzed according to pediatric nurses' general characteristics (Table 4), the average knowledge score was higher among nurses with a master's degree or higher $(9.4 \pm 1.6)$ than among those with a professional college degree or a bachelor's degree $(8.0 \pm 1.8)(\mathrm{t}=2.43, p=.017)$. The knowledge scores of nurses who worked in neonatal units $(8.6 \pm 1.4)$ were meaningfully higher than those of nurses who worked in other departments $(7.9 \pm 1.9)(t=2.42 p=.017)$. K-ICPR scores were higher among those who had experience raising children than among those who did not $(\mathrm{t}=2.34, p=$ .021) and among those who had more than 5 years of clinical experience than among those with less than 5 years of experience $(t=3.39, p=.001)$.

2) Pediatric nurses' characteristics and SUID-related confidence When confidence levels were analyzed by pediatric nurses' characteristics, it was found that nurses who had experience raising children $(\mathrm{t}=4.30, p<.001)$, had more than 5 years of clinical experience $(t=2.77, p=.006)$, had a master's level education or above $(t=2.50, p=.014)$, and had been educated about SUID ( $t=5.00, p<.001)$ showed greater confidence in educating parents about a safe sleep environment than their counterparts (Table 4).
3) Associations with conducting parental education on safe sleep environments

Significant relationships were found between whether participants delivered parental education according to unit of service $\left(x^{2}=6.69, p=.010\right)$ and SUID-related education experience $\left(x^{2}=9.17, p=.002\right)$ (Table 4).

\section{DISCUSSION}

In order to reduce the risk of SUID, providing a safe sleep environment and responding appropriately to any unexpected events are important. Pediatric nurses have the responsibility to provide related knowledge to infants' parents. This study investigated pediatric nurses' scores on the novel $\mathrm{K}$ SSSP and K-ICPR scales and factors that influenced these knowledge areas. The main findings are discussed in this section.

First, the average percentage of correct responses on the K-SSSP among pediatric nurses was $62.6 \%$, which is lower than the safe sleep environment knowledge scores that have been reported for nurses from other countries. In a US study on safe sleep practices related to infant sleep death, Barsman et al. [24] reported that the average percentage of correct responses was $74.6 \%$, while Grazel et al. [22] reported that $85 \%$ of NICU nurses correctly differentiated a safe sleep environment from an unsafe sleep environment. In countries such as Australia and the United States, SUID case management and education are overseen rigorously, and research on SUID and safe sleep environments has been active since the early 1990s [7]. In a number of previous studies, pediatric nurses were found to recognize the AAP guidelines sufficiently [22]. However, limited public promotion or professional education on infant safe sleep practices has been reported at pediatric health care clinics in South Korea. This explains our finding of relatively low K-SSSP scores, along with the lack of studies measuring nurses' knowledge of safe sleep environments.

When factors related to knowledge were analyzed, the average score of the K-SSSP was significantly higher among nurses who had a master's degree or higher or who worked in

Table 3. Pediatric Nurse' Knowledge by Confidence in Education on SUID-related Safe Sleep Practices and Delivery of Education on SUID-related Safe Sleep Practices $(N=136)$

\begin{tabular}{|c|c|c|c|c|c|}
\hline \multirow{2}{*}{ Variables } & \multirow{2}{*}{ Categories } & \multicolumn{2}{|c|}{ K-SSSP } & \multicolumn{2}{|c|}{ K-ICPR } \\
\hline & & $\mathrm{M} \pm \mathrm{SD}$ & $\mathrm{r}(p)$ & $\mathrm{M} \pm \mathrm{SD}$ & $\mathrm{r}(p)$ \\
\hline \multirow[t]{3}{*}{ Delivery of SSSP education } & No & $8.0 \pm 1.8$ & & $3.1 \pm 1.0$ & \\
\hline & Yes & $8.8 \pm 1.6$ & & $3.6 \pm 0.6$ & \\
\hline & $\mathrm{t}(p)$ & $1.79(.075)$ & & $2.25(.026)$ & \\
\hline \multicolumn{2}{|l|}{ Confidence in SSSP education } & & $.22(.012)$ & & $.23(.009)$ \\
\hline
\end{tabular}

K-ICPR, knowledge of infant cardiopulmonary resuscitation; K-SSSP, knowledge of sudden unexplained infant death related safe sleep practices; SSSP, sudden unexplained infant death related safe sleep practices. 
Table 4. Comparison of Knowledge, Confidence in Education on SUID-related Safe Sleep Practices and Delivery of Education on SUID-related Safe Sleep Practices by Nurses' Characteristics $(N=136)$

\begin{tabular}{|c|c|c|c|c|c|c|c|c|c|c|c|}
\hline \multirow{3}{*}{ Variables } & \multirow{3}{*}{ Categories } & \multirow{3}{*}{$\mathrm{n}$} & \multicolumn{2}{|c|}{ K-SSSP } & \multicolumn{2}{|c|}{ K-ICPR } & \multicolumn{2}{|c|}{$\begin{array}{l}\text { Confidence in } \\
\text { SSSP education* }\end{array}$} & \multicolumn{3}{|c|}{ Delivery of SSSP education } \\
\hline & & & \multirow{2}{*}{$\mathrm{M} \pm \mathrm{SD}$} & \multirow{2}{*}{$\mathrm{t}(p)$} & \multirow{2}{*}{$\mathrm{M} \pm \mathrm{SD}$} & \multirow{2}{*}{$\mathrm{t}(p)$} & \multirow{2}{*}{$\mathrm{M} \pm \mathrm{SD}$} & \multirow{2}{*}{$\mathrm{t}(p)$} & No & Yes & \multirow{2}{*}{$x^{2}(p)$} \\
\hline & & & & & & & & & $\mathrm{n}(\%)$ & $\mathrm{n}(\%)$ & \\
\hline Total score & & 136 & $8.1 \pm 1.8$ & & $3.1 \pm 1.0$ & & $2.6 \pm 0.9$ & & 118 & 18 & \\
\hline \multirow{2}{*}{$\begin{array}{l}\text { Having own } \\
\text { child(ren) }\end{array}$} & No & 87 & $8.1 \pm 1.7$ & 0.82 & $3.0 \pm 1.0$ & 2.34 & $2.4 \pm 0.9$ & 4.30 & $77(65.3)$ & $10(55.6)$ & 0.64 \\
\hline & Yes & 49 & $8.3 \pm 1.9$ & $(.414)$ & $3.4 \pm 0.9$ & $(.021)$ & $3.0 \pm 0.9$ & $(<.001)$ & $41(34.7)$ & $8(44.4)$ & $(.425)$ \\
\hline \multirow{2}{*}{$\begin{array}{l}\text { Clinical } \\
\text { experience }\end{array}$} & $<5$ years & 64 & $8.0 \pm 1.7$ & 0.86 & $2.8 \pm 1.0$ & 3.39 & $2.4 \pm 0.9$ & 2.77 & $55(46.6)$ & $9(50.0)$ & 0.07 \\
\hline & $\geq 5$ years & 72 & $8.3 \pm 1.8$ & $(.389)$ & $3.4 \pm 0.9$ & $(.001)$ & $2.8 \pm 0.9$ & $(.006)$ & $63(53.4)$ & $9(50.0)$ & $(.788)$ \\
\hline \multirow{2}{*}{$\begin{array}{l}\text { Level of } \\
\text { education }\end{array}$} & Associate or bachelor's degree & 125 & $8.0 \pm 1.8$ & 2.43 & $3.1 \pm 1.0$ & 0.51 & $2.6 \pm 0.9$ & 2.50 & $109(92.4)$ & $16(88.9)$ & $0.26^{\dagger}$ \\
\hline & Master's degree or higher & 11 & $9.4 \pm 1.6$ & $(.017)$ & $3.3 \pm 1.0$ & $(.611)$ & $3.3 \pm 0.6$ & $(.014)$ & $9(7.6)$ & 2 (11.1) & $(.640)$ \\
\hline \multirow{2}{*}{$\begin{array}{l}\text { Received } \\
\text { education } \\
\text { on SUID }\end{array}$} & No & 60 & $7.8 \pm 1.8$ & 1.90 & $3.0 \pm 1.0$ & 1.83 & $2.2 \pm 0.8$ & 5.00 & $58(49.2)$ & $2(11.1)$ & 9.17 \\
\hline & Yes & 76 & $8.4 \pm 1.7$ & $(.059)$ & $3.3 \pm 1.0$ & $(.069)$ & $3.0 \pm 0.9$ & $(<.001)$ & $60(50.8)$ & $16(88.9)$ & $(.002)$ \\
\hline \multirow{2}{*}{$\begin{array}{l}\text { Unit of } \\
\text { service }\end{array}$} & NICU, nursery & 53 & $8.6 \pm 1.4$ & 2.42 & $3.2 \pm 1.0$ & 0.95 & $2.8 \pm 1.0$ & 1.44 & 41 (34.7) & $12(66.7)$ & $6.69^{\dagger}$ \\
\hline & Others $^{\dagger}$ & 83 & $7.9 \pm 1.9$ & $(.017)$ & $3.1 \pm 1.0$ & $(.346)$ & $2.5 \pm 0.9$ & $(.153)$ & $77(65.3)$ & $6(33.3)$ & $(.010)$ \\
\hline
\end{tabular}

${ }^{*}$ Missing data were not included; ${ }^{\dagger}$ Fisher's exact test; ${ }^{\dagger}$ Pediatric ward, obstetric ward, pediatric emergency room, pediatric outpatient department, delivery room; K-ICPR, knowledge of infant cardiopulmonary resuscitation; K-SSSp, knowledge of sudden unexplained infant death-related safe sleep practices; NICU, neonatal intensive care unit; SSSP, sudden unexplained infant death related safe sleep practices, SUID, sudden unexplained infant death.

neonatal departments. However, nurses' clinical experience and SUID-related education were not significantly associated with knowledge of safe sleep environments. The findings regarding level of education can be explained by the fact that nurses receive further structural education in their specialization after acquiring clinical experience related to children and developing a stronger interest in this issue. This result suggests that knowledge of safe sleep environments is not acquired organically through long clinical experience; instead, it results from being specifically educated on SUID and infant safe sleep practices through higher education and delivering SUID education using systemic protocols.

Second, the average percentage of correct responses for the K-ICPR was $62.5 \%$. The percentage of correct responses for each item ranged from $29.4 \%$ to $88.2 \%$. Compared to a study of 133 European cardiovascular nurses showing percentages of correct responses that ranged from $55 \%$ to $95 \%$ [25], the correct answer rate in this study seemed to be lower. In emergency situations such as asphyxiation and sudden death of infants, pediatric nurses should have correct knowledge to perform CPR in a precise manner and should be able to educate the parents of infants in the infant discharge education procedure. This finding suggests that Korean pediatric nurses may not have enough knowledge of ICPR. Thus, it is urgently necessary to enhance and promote ICPR education for pediatric nurses. In our study, the two significant factors influencing K-ICPR were clinical experience and having one's own child(ren), as K-ICPR scores were significantly higher among nurses with longer clinical experience and with experience raising children. However, nurses with longer clinical experience might also have a higher likelihood of having experienced raising their own child(ren) as a function of time. Thus, the individual effect of these two relationships should be interpreted with caution and additional research may be necessary.

Third, pediatric nurses' confidence in delivering education on a safe sleep environment and confidence in SSSP was a mean of 2.6 on a 1-5 point scale, which is considered as fairly low confidence. In the study conducted by Hodges et al. [26], $82 \%$ of nurse-midwives responded that they were confident in carrying out their work duties related to a safe environment. As well, in the study of Kacho [15], 64\% of nurses responded that they were confident when they delivered anticipatory guidance on expanded back-to- sleep guidelines and practices. These findings suggest that the nurses in our study were less confident in safe sleep practices themselves and in educating parents on this topic than nurses in Western societies. This lack, as mentioned earlier, implies that SUID research and education are generally insufficient in the domestic context. In the US and Australia, many institutions are actively conducting research to develop quality improvement programs for SUID-related education and case management, and multifaceted efforts are being made at the national level $[27,28]$. However, there is a lack of population-wide 
awareness campaigns and activism in South Korea, and no related quality improvement programs have been reported. In this study, K-SSSP scores and confidence demonstrated a weak positive correlation, and the level of confidence was higher among nurses who had received SUID education, had a master's degree or higher, had experience raising their own children, and had longer clinical experience. Therefore, when discussing pediatric nurses' confidence related to safe sleep environments, it is difficult to ignore the influence of knowledge. For this reason, an education program that aims to increase professional SUID-related knowledge among pediatric nurses should be developed.

Fourth, $55.9 \%$ of participants had received education related to SUID, but only $13.2 \%$ delivered SSSP education to parents. This percentage itself is significantly lower than the figures found in US studies, where 73\% of NICU nurses [22] and $79 \%$ of nursery nurses [29] conducted education on SUID as a part of the discharge process. As well, this finding implies that even though some nurses might have been taught about SUID, most of them seemed not to provide SSSP education to infant parents. The main reason for this might be the lack of an adequate protocol for SUID and SSSP education in pediatric clinics in Korea. Pediatric nurses might receive some education about SUID and SSSP; however, without a concrete protocol and the system to deliver that education in a standardized manner, it might be difficult for pediatric nurses to gain enough knowledge and to carry out parental education. Therefore, there is an urgent need to educate pediatric nurses on SUID and SSSP, to develop a related educational program, and to establish a systemic protocol to provide that education as a standard component of patient safety at pediatric clinics in Korea.

Furthermore, only $5.9 \%$ of nurses responded that their wards had SSSP guidelines. A previous study in Turkey showed that $42 \%$ of nurses stated that their institutions had prepared SUID prevention guidelines, and $47 \%$ did not know about the presence of guidelines [29]. In light of previous studies reporting that when systematic guidelines were prepared at an institution or ward, parental education was delivered 4.3 times more frequently and nurses' knowledge level increased compared to counterparts, there is a need to develop clear guidelines that can be used to educate caretakers in the South Korean nursing setting [23].

It was found that nurses working in neonatal departments delivered parental education more frequently than nurses in other departments. SUID is most often experienced by infants younger than 6 months and often occurs in their sleep; therefore, nurses in neonatal units and parents both recognize the importance of SUID and safe sleep environments and have a greater need for education. However, there is a need to vital- ize SUID-related safe sleep education in other pediatric departments such as pediatric wards, pediatric intensive care units, and pediatric outpatient departments. In this study, no statistically significant association was found between pediatric nurses' knowledge of safe sleep environments and the delivery of parental education. This result contradicts those of previous reports that nurses with greater SUID-related knowledge were 1.92 times more likely to discuss SUID and safe sleep environments with infants' parents than those with lower SUID-related knowledge [30]. The contradiction can be attributed to the fact that conducting parental education is influenced not only by the educators' level of knowledge, but also by a complex combination of environmental factors such as institutional policies and the existence of educational materials, as well as individual factors such as educators' attitudes toward their safe sleep environment responsibilities. Along with efforts to increase pediatric nurses' knowledge level through professional education, it is necessary to hold discussions for parental education to actually be implemented at the organizational and departmental levels.

One of the limitations of this study is that it only included clinical nurses working at hospitals. Infants younger than 6 months are susceptible to SUID, and regardless of the presence of an underlying disease, most die at home during sleep. Therefore, it is necessary to conduct further research among pediatric nurses and nursery staff in the community. Moreover, since the data for this study were collected from a specific sample from 3 hospitals, caution is required when generalizing and interpreting the research findings. Another limitation relates to the tools used to measure the study variables: the K-SSSP, K-ICPR and VAS for confidence on SSSP education. Since there was no standardized measure for factors such as specific knowledge, we developed these tools and checked their reliability and validity. However, further research would be desirable to refine these tools with respect to standardizing psychosocial measurements in various pediatric health care settings.

\section{CONCLUSION}

This study analyzed factors related to the K-SSSP, K-ICPR, confidence in SSSP education, and clinical experiences of SSSP and ICPR. Nurses with a master's degree or higher and working in a newborn nursery had higher K-SSSP scores, and those with longer clinical experience and child-rearing experience had higher K-ICPR scores. Higher confidence levels were associated with having received SUID-related experience and having higher K-SSSP and K-ICPR scores, a master's degree or above, longer clinical experience, and experience raising children. Nurses in neonatal departments conducted parental edu- 
cation more frequently than other nurses.

These results reflect the lack of SUID-related safe sleep environment guidelines and education applicable to the nursing environment in South Korea. Therefore, this study makes the following suggestions. Since pediatric nurses have the role of educating infants' parents about SUID and safe sleep environments, systematic and professional education should be given to pediatric nurses in order to increase their knowledge and confidence levels. Moreover, in order to reduce barriers hindering parental education, educational materials such as guidelines, brochures, and videos should be developed.

\section{Conflict of interest}

No existing or potential conflict of interest relevant to this article was reported.

\section{Data availability}

Please contact the corresponding author for data availability.

\section{REFERENCES}

1. Krous HF, Beckwith B, Byard RW, Rognum TO, Bajanowski T, Corey $\mathrm{T}$, et al. Sudden infant death syndrome and unclassified sudden infant deaths: A definitional and diagnostic approach. Pediatrics. 2004;114(1):234-238.

https://doi.org/10.1542/peds.114.1.234

2. Filiano JJ, Kinney HC. A perspective on neuropathologic findings in victims of the sudden infant death syndrome: The triple-risk model. Neonatology. 1994;65(3-4):194-197.

https://doi.org/10.1159/000244052

3. Moon RY, Horne RSC, Hauck FR. Sudden infant death syndrome. Lancet. 2007;370(9598):1578-1587. https://doi.org/10.1016/S0140-6736(07)61662-6

4. Statistics Korea. Infant death and mortality [Internet]. Daejeon: Statistics Korea; 2020 [cited 2020 June 27]. Available from: http://kosis.kr/statHtml/statHtml.do?orgId=101\&tblId=DT_1B 34E08\&vw_cd=MT_ETITLE\&list_id=D11\&scrId=\&seqNo=\&lang uage=en\&obj_var_id=\&itm_id=\&conn_path=A6\&path=\%252Feng $\% 252$ Fsearch $\% 252$ FsearchList.do

5. Yang KM. The risk factors and incidences of SIDS and SUDC of Koreans [dissertation]. Seoul: Yonsei University; 2010. p. 1-54.

6. Kliegman RM, St. Geme JS, Blum NJ, Shah SS, Tasker RC, Wilson KM. Nelson textbook of pediatrics. 21nd ed. Amsterdam: Elsevier; 2020. p. 2167.

7. Task Force on Sudden Infant Death Syndrome. SIDS and other sleep-related infant deaths: Updated 2016 recommendations for a safe infant sleeping environment. Pediatrics. 2016;138(5):e20162938. https://doi.org/10.1542/peds.2016-2938
8. Ely DM, Driscoll AK. Infant mortality in the United States, 2017: Data from the period linked birth/infant death file [Internet]. National Vital Statistics Reports. Hyattsville, MD: National Center for Health Statistics; 2019 August [cited 2020 June 27]. Report No.: 2019-1120. Available from: https://stacks.cdc.gov/view/cdc/80304

9. Korea Centers for Disease Control and Prevention. Sudden infant death syndrome prevention leaflet [Internet]. Sejong: Ministry of Health and Welfare; 2007 [cited 2020 September 6]. Available from: http://www.cdc.go.kr/gallery.es? mid=a20503020000\&bid=0003 \&act=view\&list_no=136954

10. The Seoul Healthy First Step Project. Leaflet on how to reduce the risk of sudden infant death [Internet]. Seoul: Seoul Metropolitan Government; 2016 [cited 2020 September 6]. Available from: https://ourbaby.seoul.kr/_skin/kor/file/12_sudden_death.pdf

11. National Action Partnership to Promote Safe Sleep a Wake-Up Call to Safeguard Sleeping Infants (NAPPSS). About NAPPSS [Internet]. Boston, MA: National Institute for Children's Health Quality; 2019 [cited 2020 March 10]. Available from: http://www.nappss.org

12. Colson ER, Bergman DM, Shapiro E, Leventhal JH. Position for newborn sleep: Associations with parents' perceptions of their nursery experience. Birth. 2001;28(4):249-253.

https://doi.org/10.1046/j.1523-536X.2001.00249.x

13. Pierick TA, Van Waning N, Patel SS, Atkins DL. Self-instructional CPR training for parents of high risk infants. Resuscitation. 2012; 83(9):1140-1144. https://doi.org/10.1016/j.resuscitation.2012.02.007

14. Patton C, Stiltner D, Wright KB, Kautz DD, Ikuta L, Zukowsky K. Do nurses provide a safe sleep environment for infants in the hospital setting? An integrative review. Advances in Neonatal Care. 2015;15(1):8-22. https://doi.org/10.1097/ ANC.0000000000000145

15. Kacho AM. An investigation of the nurse pracitioner's knowledge and self-efficacy on expanded Back to Sleep Guidelines and practice of providing anticipatory guidance: A descriptive correlational study [master's thesis]. Cedarville, $\mathrm{OH}$ : Cedarville University. 2015. p. 1-28. https://doi.org/10.15385/tmsn.2015.3

16. Duncan JR, Byard RW. SIDS sudden infant and early childhood death: The past, the present and the future. Adelaide: University of Adelaide Press; 2018. p. 15-50, 169-186.

17. Ahn YM, Yang KM, Ha HI, Cho JA. Risk factors for sudden infant death syndrome and sleeping practices in Korea. Child Health Nursing Research. 2020;26(1):82-89. https://doi.org/10.4094/chnr.2020.26.1.82

18. Duff JP, Topjian AA, Berg MD, Chan M, Haskell SE, Joyner Jr BL, et al. 2019 American Heart Association focused update on pediatric advanced life support: An update to the American Heart Association guidelines for cardiopulmonary resuscitation and emergency cardiovascular care. Circulation. 2019;140(24):e904-e914. https://doi.org/10.1161/CIR.0000000000000731 
19. Korean Association of CardioPulmonary Resuscitation. Korean basic life support, 2018 [Internet]. Seoul: Korean Association of CardioPulmonary Resuscitation; 2018 September [cited 2020 September 6]. p. 1-134. Available from: http://www.kacpr.org/

20. Bienkowski P, Zatorski P, Glebicka A, Scinska A, KurkowskaJastrzebska I, Restel M, et al. Readiness visual analog scale: A simple way to predict post-stroke smoking behaviour. International Journal of Environmental Research and Public Health. 2015;12(8): 9536-9541. https://doi.org/10.3390/ijerph120809536

21. Hawker GA, Mian S, Kendzerska T, French M. Measures of adult pain: Visual analog scale for pain (VAS pain), numeric rating scale for pain (NRS pain), mcgill pain questionnaire (MPQ), short-form mcgrill pain questionnaire (SF-MPQ), chronic pain grade scale (CPGS), short form-36 bodily pain scale (SF-36 BPS), and measure of intermittent and constant osteoarthritis pain (ICOAP). Arthritis Care and Research. 2011;63(Suppl 11):S240-S252.

https://doi.org/10.1002/acr.20543

22. Grazel R, Phalen AG, Polomano RC. Implementation of the American Academy of Pediatrics recommendations to reduce sudden infant death syndrome risk in neonatal intensive care units: An evaluation of nursing knowledge and practice. Advances in Neonatal Care. 2010;10(6):332-342.

https://doi.org/10.1097/ANC.0b013e3181f36ea0

23. Aris C, Stevens TP, Lemura C, Lipke B, McMullenS, Cote-Arsenault $\mathrm{D}$, et al. NICU nurses' knowledge and discharge teaching related to infant sleep position and risk of SIDS. Advances in Neonatal Care. 2006;6(5):281-294. https://doi.org/10.1016/j.adnc.2006.06.009

24. Barsman SG, Dowling DA, Damato EG, Czeck P. Neonatal nurses' beliefs, knowledge, and practices in relation to sudden infant death syndrome risk-reduction recommendations. Advances in Neonatal Care. 2015;15(3):209-219.

https://doi.org/10.1097/ANC.0000000000000160

25. Pettersen TR, Martensson J, Axelsson A, Jorgensen M, Stromberg A, Thompson DR, et al. European cardiovascular nurses' and allied professionals' knowledge and practical skills regarding cardiopulmonary resuscitation. European Journal of Cardiovascular Nursing. 2018;17(4):336-344.

https://doi.org/10.1177/1474515117745298

26. Hodges NL, Anderson SE, McKenzie LB, Katz ML. Certified nursemidwives' knowledge, attitudes, and behaviors about infant safe sleep. Journal of Midwifery and Women's Health. 2018;63(2):196204. https://doi.org/10.1111/jmwh.12706

27. Rowe AD, Sisterhen LL, Mallard E, Borecky B, Schmid B, Rettiganti $\mathrm{M}$, et al. Integrating safe sleep practices into a pediatric hospital: Outcomes of a quality improvement project. Journal of Pediatric Nursing. 2016;31(2):e141-e147. https://doi.org/10.1016/j.pedn.2015.10.015

28. Goodstein MH, Bell T, Krugman SD. Improving infant sleep safety through a comprehensive hospital-based program. Clinical Pediatrics. 2015;54(3):212-221. https://doi.org/10.1177/0009922814566928

29. Bartlow KL, Cartwright SB, Shefferly EK. Nurses' knowledge and adherence to sudden infant death syndrome prevention guidelines. Pediatric Nursing. 2016;42(1):7-13.

30. Hodges NL, Anderson SE, McKenzie LB, Katz ML. Infant safe sleep: A survey of the knowledge, attitudes, and behaviors of obstetric physicians. Journal of Community Health. 2018;43(3):488-495. https://doi.org/10.1007/s10900-017-044 